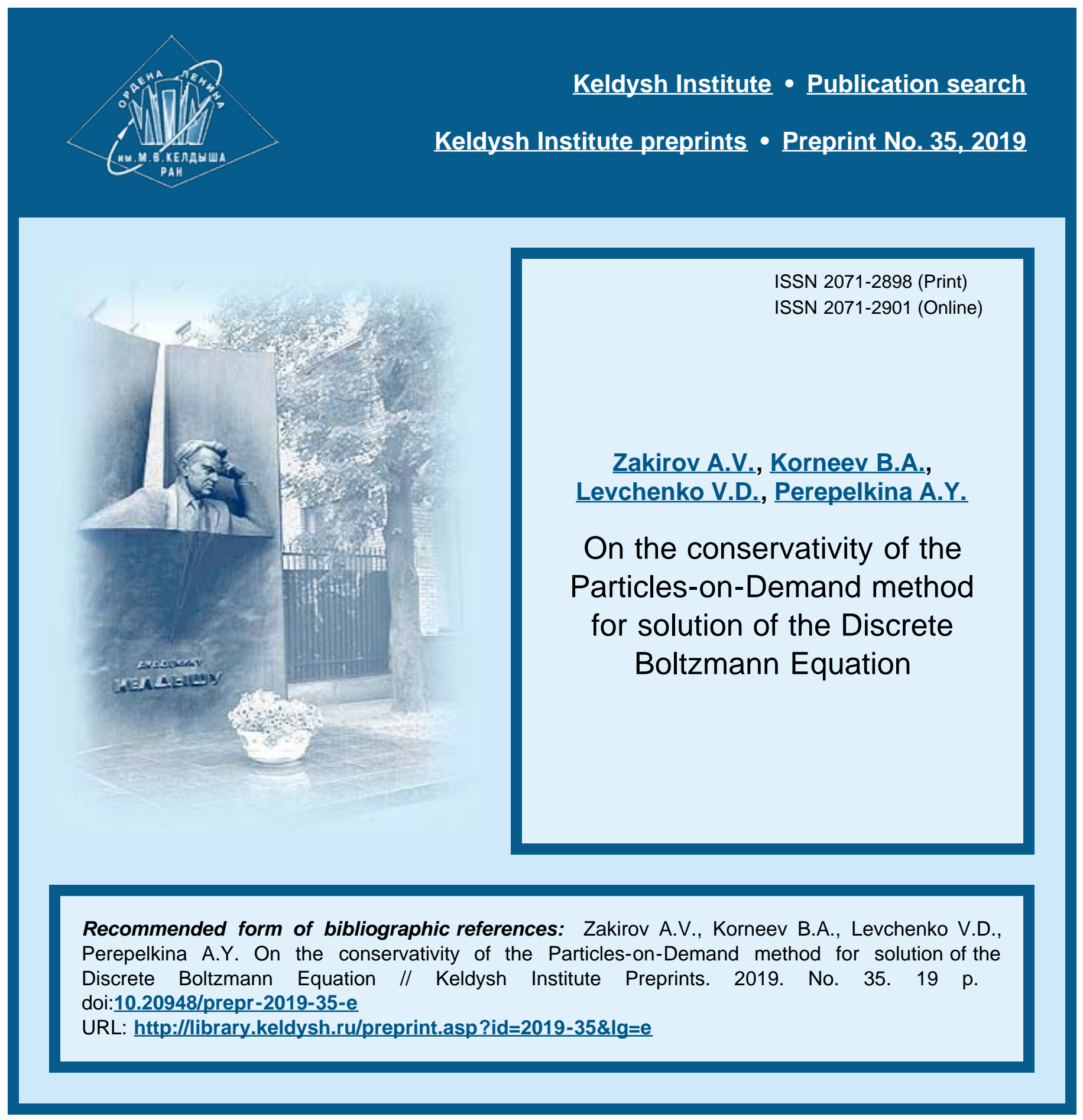




\author{
Ордена Ленина \\ ИНСТИТУТ ПРИКЛАДНОЙ МАТЕМАТИКИ \\ имени М. В. Келдыша \\ Российской академии наук
}

Andrey Zakirov, Boris Korneev, Vadim Levchenko, Anastasia Perepelkina

\title{
On the conservativity of the Particles-on-Demand method for the solution of the Discrete Boltzmann Equation
}




\section{А.В. Закиров, Б.А. Корнеев, В.Д. Левченко, А.Ю. Перепёлкина \\ K вопросу о консервативности метода PonD решения дискретного уравнения Больцмана}

Метод решёточного уравнения Больцмана (LBM) с успехом применяется при решении задач динамики жидкостей и газов в области малых скоростей потока и при изотермических условиях. Недавно был предложен альтернативный метод PonD решения дискретного уравнения Больцмана, свободный от указанных ограничений. В данной работе представлена собственная реализация данного метода и исследованы возможности решения с его помощью задач с ударными волнами. В вычислительном аспекте, по сравнению со стандартным подходом LBM, шаг столкновений упрощается, но для выполнения переноса требуется применять неявную схему, вычислительно сложную и неконсервативную в общем случае. Мы предлагаем вариант метода PonD, обеспечивающий консервативность и использующий явную схему переноса. Выполнение законов сохранения массы, импульса и энергии проверено в задаче формирования слабой ударной волны при опрокидывании акустической волны большой амплитуды. Также представлено решение 1D и 3D проблем Сода. Ключевые слова: метод решёточного уравнения Больцмана, дискретное уравнение Больцмана, консервативность численных схем

\section{A. Zakirov, B. Korneev, V. Levchenko, A. Perepelkina}

On the conservativity of the Particles-on-Demand method for the solution of the Discrete Boltzmann Equation

It is well known that the standard Lattice-Boltzmann method (LBM) is applicable in the range of small flow velocities and under the isothermal conditions. The novel Particle-on-demand method [1] allows to numerically solve the discrete Boltzmann equation for high Mach numbers. We validate its capabilities with our implementation on the problems with shock waves. In comparison with the standard Lattice Boltzmann Method, the collision step is simple, but the streaming step is implicit, non-conservative and excessively computationally heavy. We propose a way that in specific cases improves the method by making the streaming step explicit and conservative. The results are validated by examining the total mass, momentum and energy change in the problem of shock formation due to the sound wave distortion. The scheme also performs well in both 1D and 3D test Sod problems.

Keywords: Lattice-Boltzmann method, high Mach number, conservation property of numerical schemes 


\section{Introduction}

It is well known that the standard Lattice-Boltzmann method (LBM) [2] is applicable in the range of small flow velocities and under the isothermal conditions. The temperature, and zero flow velocity, are ingrained in the integration variable of the Gaussian quadrature [3]. If the flow speed is comparatively large, the Galilean transformation allows to measure flow speed as a deviation from the reference one, and thus the applicability conditions remain valid.

In view of this, the ability to change the reference frame of the solution with spatial coordinate seems a tempting idea in the problems with large variations of speed and temperature. However, it is forbidden in the standard methods since the Lattice-Boltzmann Equation (LBE) is written is terms of the discrete distribution functions $f_{i}$, which correspond to the expansion of the continuous distribution function around the reference value [3]. Thus, the change of the reference frame would mean the change of variables [4], and this fact restricts the change of the reference frame.

The solution was proposed in [1] by construction of the Particles-on-Demand (PonD) LBM which belongs to the family of semi-Lagrangian methods of LBM [5, 6]. Indeed, there is an elegant way to change the gauge of the LBM variables by equating the moments. The change of gauge comes down to the change of the integration variable.

The results reported in [1] show perfect match with the theoretical prediction up to Mach numbers of 100 . It is very interesting to investigate the ability of this method for modeling discontinuities. In this work we implement the scheme and test its applicability to the simulation of strong shock waves.

The weak point of the proposed method is the complexity of the streaming step. The streaming step requires off-grid values of the discrete distribution functions. This is often avoided in LBM simulations. The original LBM streaming is simple for implementation and does not lead to numerical dispersion. The necessity of the off-grid values arises in the simulations with non-cartesian grids [7] or with grid refinement [8, 9]. Moreover, the streaming step in [1] becomes implicit and requires predictor-corrector iterations.

As in other interpolation-supplemented LBMs, the issue is whether or not the conservation laws hold for the given scheme, and if not, whether it is possible to complement the simulation in a way that the scheme becomes conservative. 


\section{D1Q5 Particles-on-Demand Lattice-Boltzmann method}

\subsection{Velocity space discretization of the distribution function}

The dicrete Boltzmann equation with collision term in the BGK form reads

$$
\frac{\partial f_{i}}{\partial t}+\mathbf{c}_{i} \frac{\partial f_{i}}{\partial \mathbf{r}}=\frac{f_{i}-f_{i}^{e q}}{\tau} .
$$

Here $f_{i}$ are the Discrete Distribution Functions (DDF). They are used to compute an approximation to the moments $\int f(\mathbf{v}, \mathbf{r}, t) v_{x}^{\alpha} v_{y}^{\beta} v_{z}^{\gamma} d \mathbf{v}$ of the continuous distribution function $f(\mathbf{v}, \mathbf{r}, t)$ with numerical integration by Gaussian quadrature rule:

$$
m_{\alpha \beta \gamma} \equiv \int f^{N}(\mathbf{v}, \mathbf{r}, t) v_{x}^{\alpha} v_{y}^{\beta} v_{z}^{\gamma} d \mathbf{v}=\sum f_{i} c_{i x}^{\alpha} c_{i y}^{\beta} c_{i z}^{\gamma},
$$

where $f^{N}(\mathbf{v}, \mathbf{r}, t)$ is an expansion of the continuous DF into Hermite polynomial basis, truncated in order $N$.

When the quadrature with the order of approximation $n$ is taken, that is, the quadrature is exact for polynomials up to order $n$, equation (2) is exact for $\alpha+\beta+\gamma+N<n$ and

$$
f_{i}=w_{i} f\left(\mathbf{c}_{i}, \mathbf{r}, t\right)(2 \pi)^{D / 2} \exp \left(\left|\mathbf{c}_{i}\right|^{2} / 2\right),
$$

$w_{i}$ and $c_{i}$ are the weights and abscissa of the Gaussian quadrature.

$$
(2 \pi)^{D / 2} \exp \left(\left|\mathbf{c}_{i}\right|^{2} / 2\right)
$$

is the inverse of the weight function of the Hermite polynomials, and, at the same time, of the Maxwell distribution function for temperature $T=1$ and flow velocity $\mathbf{u}=0 . f_{i}^{e q}$ corresponds to the equilibrium distribution function and is usually taken in form of a polynomial.

For example, in one-dimensional case $(D=1)$, for the quadrature with the order of accuracy equal to 5 , the lowest number of the required abscissa is three: $(0, \pm \sqrt{3})$. With the change of the integration variable the abscissa are usually scaled to match the grid spacing. In this case, $\mathbf{c}_{i}$ are in $(0, \pm 1)$ and the scaling introduces the "lattice temperature" $T_{0}=1 / \sqrt{3}$.

In the PonD method the integration variable is scaled by an arbitrary temperature $T$ and shifted by an arbitrary reference velocity $\mathbf{u}$. It becomes $\mathbf{e}_{\mathbf{i}}=\sqrt{T / T_{0}} \mathbf{c}_{\mathbf{i}}+\mathbf{u}$.

The $f_{i}$ variables are transformed with the use of the requirement that the moments should be gauge-invariant. To convert a set of $\vec{f}_{i}^{\lambda}$ from one gauge 
$\lambda(\mathbf{u}, T)$ to another gauge $\lambda^{\prime}\left(\mathbf{u}^{\prime}, T^{\prime}\right)$ the following equation is solved:

$$
M_{\lambda^{\prime}} \vec{f}_{i}^{\lambda^{\prime}}=M_{\lambda} \vec{f}_{i}^{\lambda}
$$

where the matrices $M_{\lambda}$ and $M_{\lambda^{\prime}}$ are moments calculation operators.

For the node update at $\mathbf{r}_{p}$, all $f_{i}$ should be streamed from the positions $\mathbf{r}_{p}-\mathbf{e}_{i}(\lambda)$, where $(\lambda)$ is the gauge that is obtained after this streaming update. The $f_{i}$ at the required coordinates are not known.

In regular LBM, various methods exist for space-time discretization in case the quadrature points do not correspond with the lattice points, such as various kinds of finite-difference or finite-volume methods [10, 9, 11]. As a simple example, $f_{i}$ may be interpolated from the nearby nodes with Lagrange polynomial interpolation. In [1] 4-point interpolation is used. The 4 points are found relative to the $\mathbf{r}_{p}-\mathbf{e}_{i}(\lambda)$ position, so it may vary for different $e_{i}$ in one node, and for different points.

Before the interpolation, the $f_{i}$ should be transformed to the gauge, corresponding to $(\lambda)$ at $x_{p}$ after this streaming update. Thus, in contrast to the traditional LBM, the streaming step has become implicit, computationally heavy, and generally not conservative. On the other hand, the collision step is greatly simplified, since in the defined gauge $f_{i}^{e q}=w_{i} \rho$.

\subsection{D1Q5 PonD}

First, consider $D=1$ case, with five $\vec{f}_{i}$.

Matrix $M_{\lambda}$ elements are $m_{i \alpha}=e_{i}^{\alpha}$, where $\mathbf{e}_{\mathbf{i}}=\sqrt{T / T_{0}} \mathbf{c}_{\mathbf{i}}+\mathbf{u}$ and $0 \leq \alpha \leq 4$. Vectors $\mathbf{c}_{i}$ are the base set of discrete velocities $\left(0, \pm c_{A}, \pm c_{B}\right)$.

Let $f_{j}^{\lambda^{\prime}}=G_{i j} f_{i}^{\lambda}$. The elements of the matrix $\hat{G}=M_{\lambda^{\prime}}^{-1} M_{\lambda}$ can be found in the following form:

$$
w_{i j} g_{i j}
$$

Here

$$
\begin{gathered}
w_{i j}= \begin{cases}\frac{T_{0}^{2}}{T^{\prime 2} c_{A}^{2} c_{B}^{2}} & \text { if } c_{j}^{\prime}=0 ; \\
\frac{T_{0}^{2}}{2 T^{\prime 2}\left(c_{A}^{4}-c_{B}^{4}\right)} & \text { if }\left|c_{j}^{\prime}\right|=c_{A} ; \\
\frac{T_{0}^{2}}{2 T^{\prime 2}\left(c_{B}^{4}-c_{A}^{4}\right)} & \text { if }\left|c_{j}^{\prime}\right|=c_{B} ;\end{cases} \\
g_{i j}=\frac{\prod_{k=0, . .4}\left(\Delta \mathbf{u}-\sqrt{T^{\prime} / T_{0}} \mathbf{c}_{k}+\sqrt{T / T_{0}} \mathbf{c}_{i}\right)}{\Delta \mathbf{u}-\sqrt{T^{\prime} / T_{0}} \mathbf{c}_{j}+\sqrt{T / T_{0}} \mathbf{c}_{i}}, \Delta \mathbf{u}=\mathbf{u}-\mathbf{u}^{\prime} .
\end{gathered}
$$


The choice of $c_{A}, c_{B}$ and $T_{0}$ is generally free. To get finer approximation for higher moments, it is better to choose them as zeros of the correspondent Hermite polynomials (i.e. 5 th order Hermite polynomials in D1Q5). Here we take $T_{0}=1, c_{A}=\sqrt{5-\sqrt{10}}, c_{B}=\sqrt{5+\sqrt{10}}$.

The weights $W_{i}$ of the equilibrium functions can be found from the following equations:

$$
\begin{aligned}
W_{0}+2 W_{A}+2 W_{B} & =1 ; \\
2 c_{A}^{2} W_{A}+2 c_{B}^{2} W_{B} & =T_{0} ; \\
2 c_{A}^{4} W_{A}+2 c_{B}^{4} W_{B} & =3 T_{0}^{2} .
\end{aligned}
$$

Solving it we get:

$$
W_{A}=\frac{T_{0}\left(c_{B}^{2}-3 T_{0}\right)}{2 c_{A}^{2}\left(c_{B}^{2}-c_{A}^{2}\right)} ; \quad W_{B}=\frac{T_{0}\left(c_{A}^{2}-3 T_{0}\right)}{2 c_{B}^{2}\left(c_{A}^{2}-c_{B}^{2}\right)} ; \quad W_{0}=1-2 W_{A}-2 W_{B} .
$$

\subsection{Explicit PonD scheme with a fixed interpolation stencil}

An interesting observation can be made if we try to express the first moments at a point in the specific case of the fixed stencil. By the fixed stencil we mean the use of the same interpolation points for all $e_{i}$ in every $x_{p}$ node, and for all $x_{p}$.

In $1 \mathrm{D}$ case, let us take $\mathrm{Q}$ discrete velocities so that $\mathrm{Q}$ independent moments can be computed. Let us take Lagrange polynominal interpolation with $L$ points. The $f_{i}$ at a point $x_{p}$ in space in the $(\mathbf{u}, T)$ gauge comes from the streaming operation from the point $x_{p}-e_{i}(u, T)$. At $e_{i}$, it is obtained by the interpolation

$$
\left.f_{i}\right|_{x_{p}-e_{i}}=\sum_{n=0}^{L-1} a_{n} f_{i}\left(x_{n}\right)
$$

$x_{p}$ is one of the points from the interpolation stencil $0<=p<=L-1$. $a_{n}$ are the coefficients of the interpolation stencil for the value at $x_{n}$ :

$$
a_{n}\left(x_{p}, e_{i}\right)=\prod_{k=0}^{L-1} \frac{x_{p}-e_{i}-x_{k}}{x_{n}-x_{k}} .
$$

This expression has $L$ factors and thus it is a polynomial in $e_{i}$ of order $L$. It may be expressed by a sum

$$
a_{n}=\sum_{l=0}^{L} A_{n l} l_{i}^{l}
$$


where $A_{n l}$ are readily found after fixing $L$ and $x_{p}$.

After streaming, the moments at $x_{p}$ are expressed as:

$$
m_{\alpha}=\sum_{i=0}^{Q-1} e_{i}^{\alpha} \sum_{n=0}^{L-1} \sum_{l=0}^{L} A_{n l} l_{i}^{l} f_{i}\left(x_{n}\right)=\sum_{n=0}^{L-1} \sum_{l=0}^{L} A_{n l} m_{\alpha+l} .
$$

Provided that the $L$ moments with the order up to $m_{\alpha+L}$ are gauge-invariant, the moment of the order $\alpha$ may be obtained with an explicit stencil from the moments in the neighboring points. For example, in D1Q5, let's manually fix the nearest 3 points for interpolation of all $5 f_{i}$. We will mark these 3 points as $P_{-}, P_{0}$ and $P_{+}$assuming the nearest left point, current point and the nearest right point.

Then it can be shown that in D1Q5 the first 3 moments after the LBM pull step can be expressed in terms of the moments in these 3 points. The new moments in the point $P_{0}$ are found in the new gauge $\lambda^{\prime}$ :

$$
m_{\alpha}\left(x_{0}, t+\Delta t\right)=\sum_{i=0 . .4} f_{i}^{\lambda^{\prime}} \mathbf{e}_{i}^{\alpha}\left(x_{0}, t+\Delta t\right) .
$$

Also by the construction of the method $m_{0}=\rho, m_{1}=\rho \mathbf{u}, m_{2}=\rho \mathbf{u}^{2}+D \rho T$. If we use Lagrange interpolation by the 3 nearest points, then the new moments are as follows:

$$
\begin{aligned}
m_{\alpha}^{0}(t+\Delta t)=m_{\alpha}^{0}(t) & -\frac{1}{2}\left(m_{\alpha+1}^{+}(t)-m_{\alpha+1}^{-}(t)\right) \\
& +\frac{1}{2}\left(m_{\alpha+2}^{+}(t)+m_{\alpha+2}^{-}(t)-2 m_{\alpha+2}^{0}(t)\right), \alpha=1,2,3,
\end{aligned}
$$

where $m^{0}, m^{-}$and $m^{+}$are the moments in the points $P^{0}, P^{-}$and $P^{+}$accordingly.

In D1Q5 method we can retrieve only the the first 5 moments, then only the first 3 moments can be expressed explicitly in accordance with eq. (14). But it is enough to extract all necessary information for the new gauge (i.e. $\mathbf{u}$ and $T$ ) and we don't need to make iterative procedure to find correct gauge. These method allows to get the new set of $\mathbf{e}_{i}$ in the new gauge. The rest of $f_{i}$ in these new gauge should be interpolated.

With help of this new procedure the original iterative implicit method becomes explicit. Note also that the formula (14) also guarantees that this numerical scheme is fully conservative in mass, momentum and energy.

The consideration is valid if the interpolation stencil for all $f_{i}$ at the node update stays fixed. It may occur naturally in the simulation, and the chances are higher if the gauge temperature is low and the sign of $u$ doesn't change. For $L=3$, if $e_{i}$ length is smaller than the half of the mesh step, the interpolation 
stencil for all $f_{i}$ is same from the start of simulation, but may become different in the course of the simulation. We may either put restrictions on the gauge parameters so that it will not happen, or enforce the fixed stencil by eliminating the dependence of the point choice on $x_{p}-e_{i}$. In the latter case, $x_{p}-e_{i}$ may fall outside the interpolation stencil if $\mathbf{e}_{i}$ becomes larger than the distance between grid nodes. This changes over the estimation from the interpolation to the extrapolation and can lead to solution fluctuation and instabilities.

\subsection{D3Q125 PonD}

The same approach is also valid in $3 \mathrm{D}$ if we make the simple direct product of all statements. Then there are $125 f_{i}$ in the single point and the transfer matrix from one gauge to another consist of the $125 \times 125$ elements. The elements are as like as (5):

$$
w_{x} w_{y} w_{z} g_{x} g_{y} g_{z}
$$

where $w_{x}$ and $g_{x}$ are constructed just as $w_{i j}$ and $g_{i j}$ in (6)-(7) but taking only $x$-component of the vectors $\mathbf{c}$ and $\mathbf{u}$, and $y$ - or $z$-component for $w_{y}, g_{y}$ and $w_{z}$, $g_{z}$.

$125 f_{i}$ also allow to construct 125 different moments $m_{\alpha \beta \gamma}=\sum_{i=0}^{124} f_{i} e_{x}^{\alpha} e_{y_{i}}^{\beta} e_{z}^{\gamma}$.

If we fix the Lagrange interpolation on the nearest points (27 neighboring nodes in $3 \mathrm{D}$ ) then it is also possible to get the explicit expression for the first moments and to find appropriate gauge.

\subsection{Conservativity}

As shown in section 2.3, the scheme is conservative if the same 3 points of the coordinate grid are chosen for the interpolation for each node update. In general 1D case the conservativity may be demonstrated with the following consideration. If a moment update in the cell $x_{j}$ may be written in the form

$$
\left.\frac{\partial m_{\alpha}}{\partial t}\right|_{x_{j}}=\left.F\right|_{x_{j}-1 / 2}-\left.F\right|_{x_{j}+1 / 2}
$$

where $F$ is some numerical representation of the flow of $m_{\alpha}$ between nodes and the integral of 16 over some region $x_{0} \ldots x_{J}$ is

$$
\left.\sum_{j=0}^{J} \frac{\partial m_{\alpha}}{\partial t}\right|_{x_{j}}=\left.F\right|_{x_{0}-1 / 2}-\left.F\right|_{x_{J}+1 / 2}
$$

where all intermediate $F$ vanish, then the moment $m_{\alpha}$ is conserved. 
In case the stencil is fixed and same for all $f_{i}$ a formula similar to 14 may be achieved, and the discrete representation of flows is evident. In other cases the left and right flows may be separated by finding the form of the moment $m_{\alpha}$ update in terms of $f_{i}$ and its sum over a sufficiently large region (larger, than the stencil width). The flows inside the summation region vanish.

However, this works only if the update rule is transitionally invariant. In case the interpolation stencil is changed inside the region, the right flow and the left flow over the cell boundary do not vanish. This becomes the source of mass, and other moments. And generally, by construction of the PonD method in [1], the stencil change of this kind is almost sure to happen during the simulation.

This shows that the method in its original form is not conservative. The mass gain will be shown on the simulation examples in later sections.

\section{Simulation examples}

\subsection{Sound wave breaking}

Here we present the test problem of sound wave breaking to demonstrate the conservativity and the differences between interpolation schemes and the number of discrete velocities.

This problem can not be solved by standard LBM, since it requires compressibility of the fluid. It may be solved in modified LBM by artificially adjusting the speed of sound [12, 13].

We test both 3D and 1D schemes, but the simulation domain is 1D with regular mesh and the space step $\Delta x=1$ and $1024 \times 1 \times 1$ cells total with periodic boundary conditions. The initial conditions are the following:

$$
\begin{aligned}
& P(x)=P_{0}+A \sin \left(x \frac{2 \pi}{256}\right) ; \\
& T(x)=T_{0} P(x)^{\frac{\gamma-1}{\gamma}} ; \\
& \rho(x)=\frac{P(x)}{T(x)} ; \\
& u(x)=\frac{A T_{0}}{P_{0} \sqrt{\gamma T_{0}}} \sin \left(x \frac{2 \pi}{256}\right) .
\end{aligned}
$$

Here $P(x), T(x), \rho(x)$ and $u(x)$ are the pressure, temperature, density and velocity. We choose the base pressure $P_{0}=1$, the base temperature $T_{0}=0.1$, amplitude $A=0.2$. Adiabatic exponent $\gamma=(D+2) / D$ is 3 in $1 \mathrm{D}$ scheme or $5 / 3$ in $3 \mathrm{D}$. 

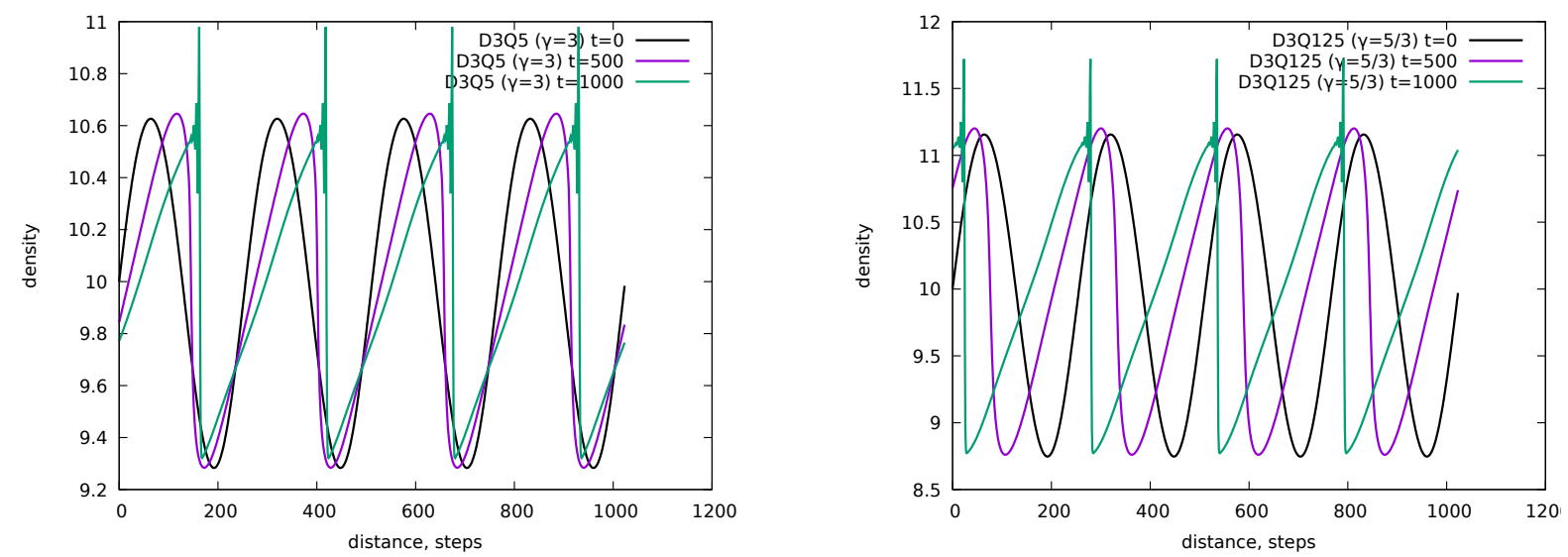

Figure 1: Sound wave density profiles at the different time moments $(t=0,500 \Delta t$ and 1000 $\Delta t$ ). The scheme is D1Q5 (left) and D3Q125 (right) with the fixed interpolation stencil using 3 nearest points.

Fig. 1 demonstrates the sinusoidal wave distortion due to the sound speed dependency on the temperature. After approximately 500 time steps, the shock wave is formed and the discontinuity of the parameters arises. The numerical scheme is non-monotonic and the oscillations begin to develop near the discontinuity.

We compare three variants of the interpolation scheme:

- 4-point interpolation stencil as in [1];

- 3-point interpolation; the center point of interpolation is found as the nearest point for every $f_{i}$ : round $\left(x_{p}-e_{i}\right)$;

- 3-point interpolation with the fixed stencil, so that the center point of the interpolation for all $f_{i}$ is $x_{p}$.

If we compare the shock wave front for different number of discrete speeds and different interpolation schemes (Fig. 2) it may be found that they are different not only in the oscillations behaviour but even in the position of the discontinuity.

Further we calculated the total mass, momentum and energy (first three moments) at all points of the computational region. All these moments must be constant in time if we use periodic boundary conditions. But Fig. 3 demonstrates that the conservativity is achieved only if we use the fixed interpolation stencil and sufficiently large set of discrete velocities ( 5 in 1D and more than 125 in 3D).

In all these cases $\tau=0.51$ and time step $\Delta t=1$. 

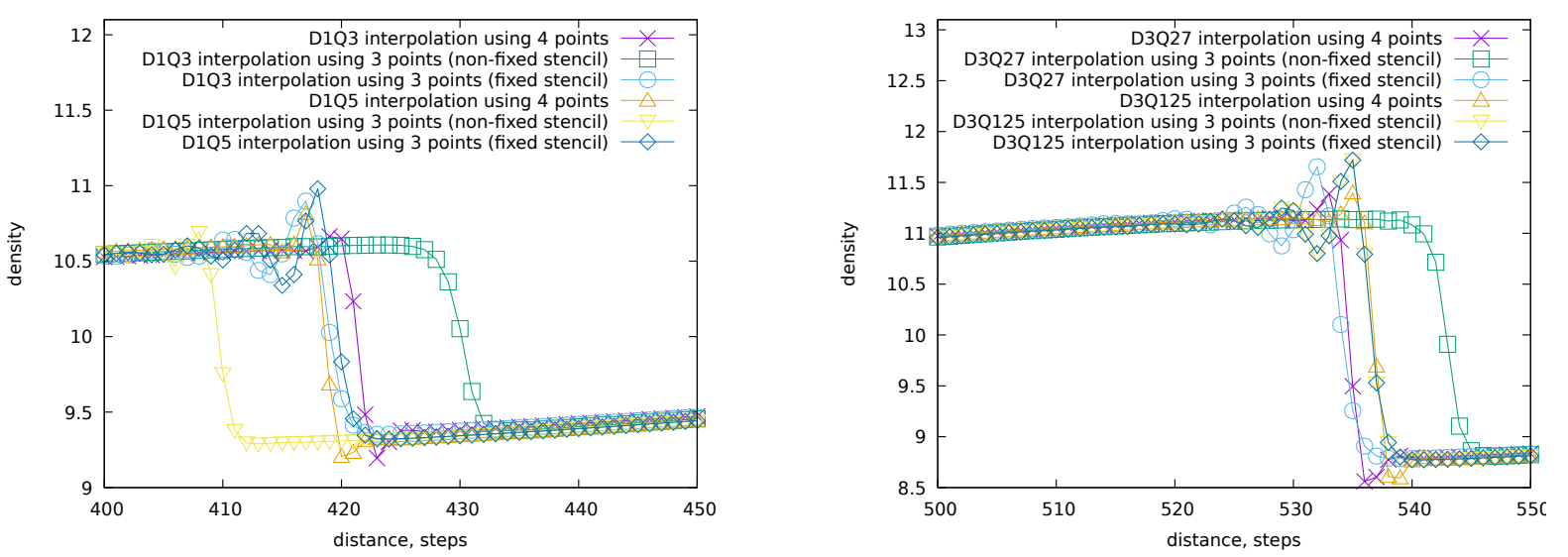

Figure 2: Shock wave profile structure after 1000 time steps for different schemes

\subsection{One-dimensional Riemann problems}

We also report the ability to solve some of the Riemann problems with PonD LBM: Sod shock tube problem [14] and the blast with a temperature ratio of $10^{5}: 1$ also known as the left part of the Woodward-Colella blast wave problem [15].

The Sod shock tube task is a common one-dimensional benchmark with the following initial conditions:

$$
\begin{aligned}
\left.\rho\right|_{x<0}=1, & \left.\rho\right|_{x \geq 0}=0.125 \\
\left.P\right|_{x<0}=0.01, & \left.P\right|_{x \geq 0}=0.001 ; \\
\left.u\right|_{x<0}=0, & \left.u\right|_{x \geq 0}=0 .
\end{aligned}
$$

We have solved it with two types of LBM PonD D1Q5: with the fixed interpolation stencil using 3 nearest points for all nodes and the flexible interpolation stencil using 4 points as described originally in [1]. The mesh is regular with $\Delta x=\Delta t=1$.

The solution after 1000 time steps is plotted in Fig. 4 .

The solution is close to exact, except for the oscillations. Moreover the oscillations are stronger for the interpolation using 3 points with the fixed stencil.

To demonstrate where the conservative scheme has the advantage we consider another Riemann problem. The second test (blast wave) has the following initial conditions:

$$
\begin{aligned}
\left.\rho\right|_{x<0}=1, & \left.\rho\right|_{x \geq 0}=1 \\
\left.P\right|_{x<0}=10^{-1}, & \left.P\right|_{x \geq 0}=10^{-6} \\
\left.u\right|_{x<0}=0, & \left.u\right|_{x \geq 0}=0 .
\end{aligned}
$$

The solution after 100 time steps (Fig. 5) can be found for D1Q5 if we use the fixed interpolation stencil using 3 nearest points. 

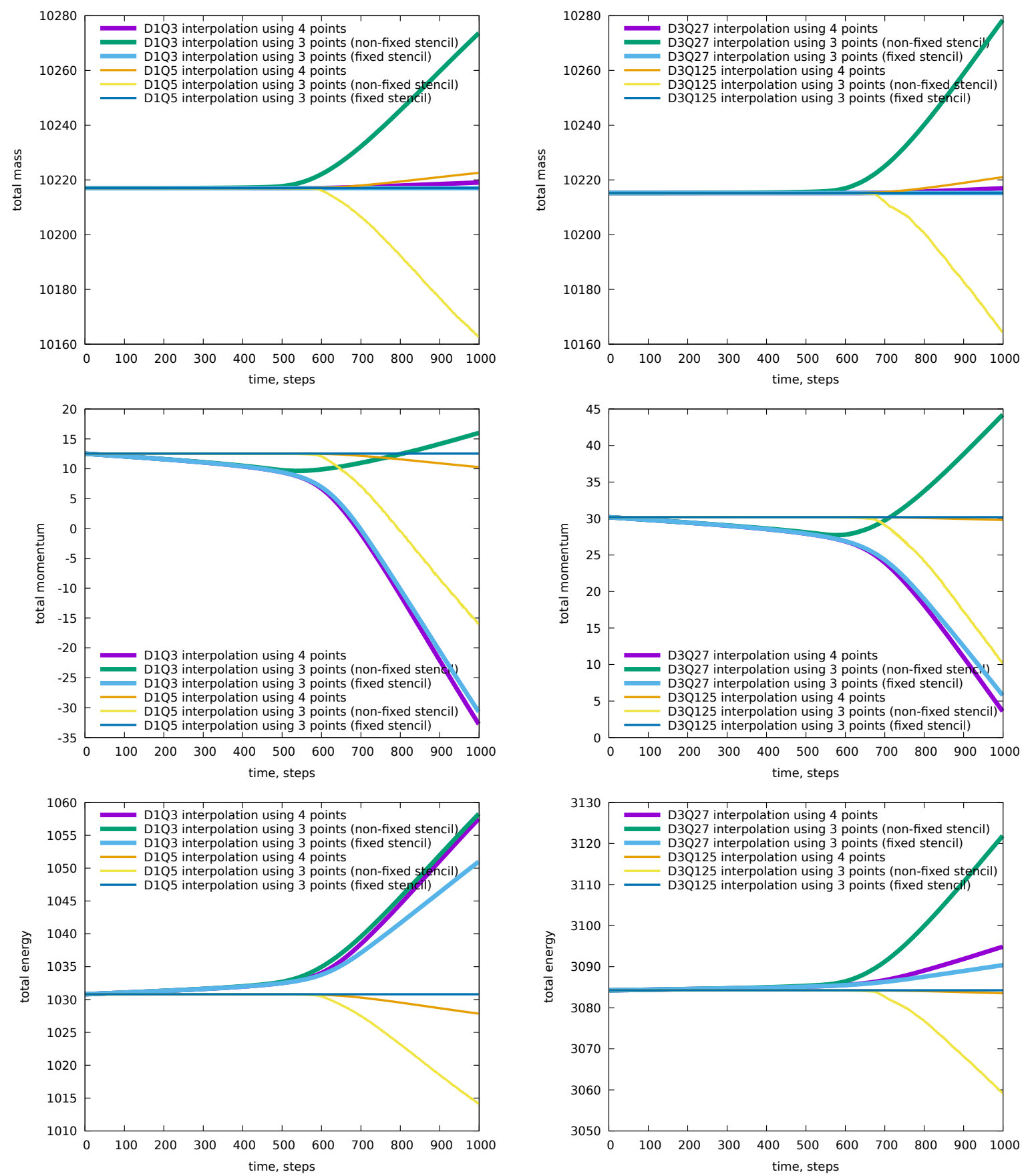

Figure 3: Total mass, momentum and energy dependency on time. 

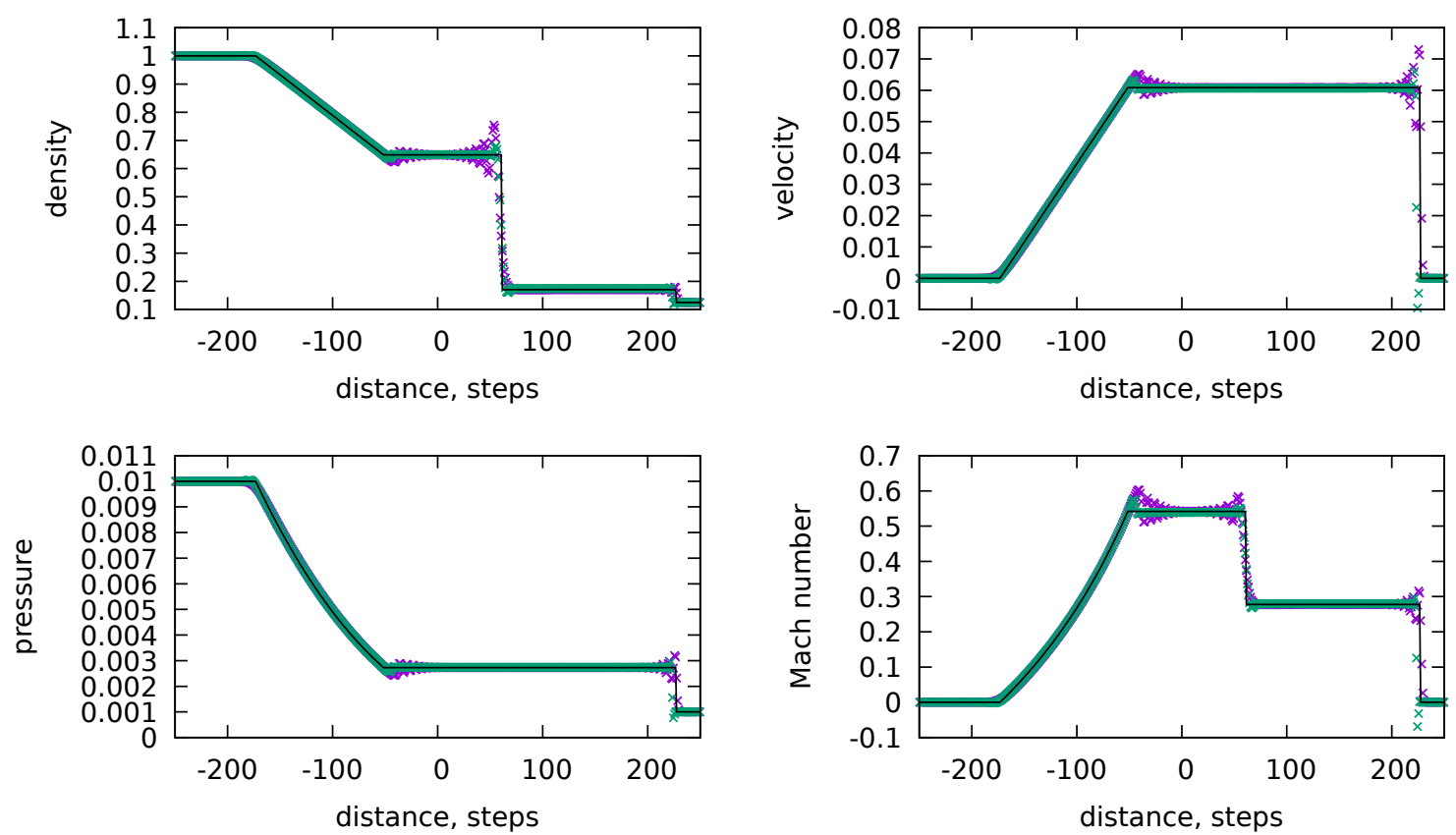

LBM PonD D105 with fixed interpolation stencil using 3 points

LBM PonD D1Q5 with interpolation over 4 points

Figure 4: Sod problem test at $1000 \Delta t, \tau=0.51$
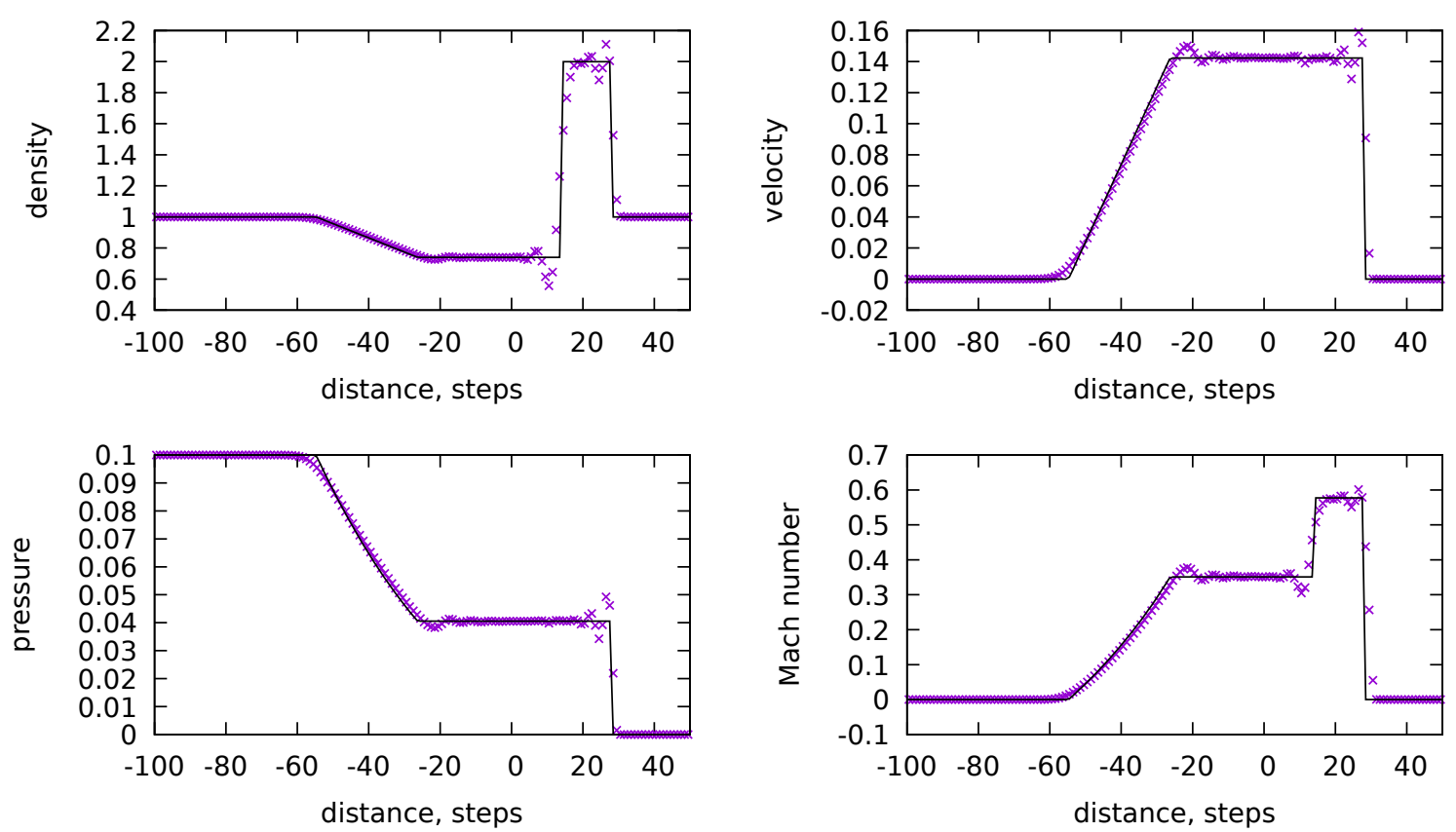

LBM PonD with fixed interpolation stencil over 3 points, $\tau=0.6$

Exact solution

Figure 5: Blast wave problem test at $100 \Delta t$ 

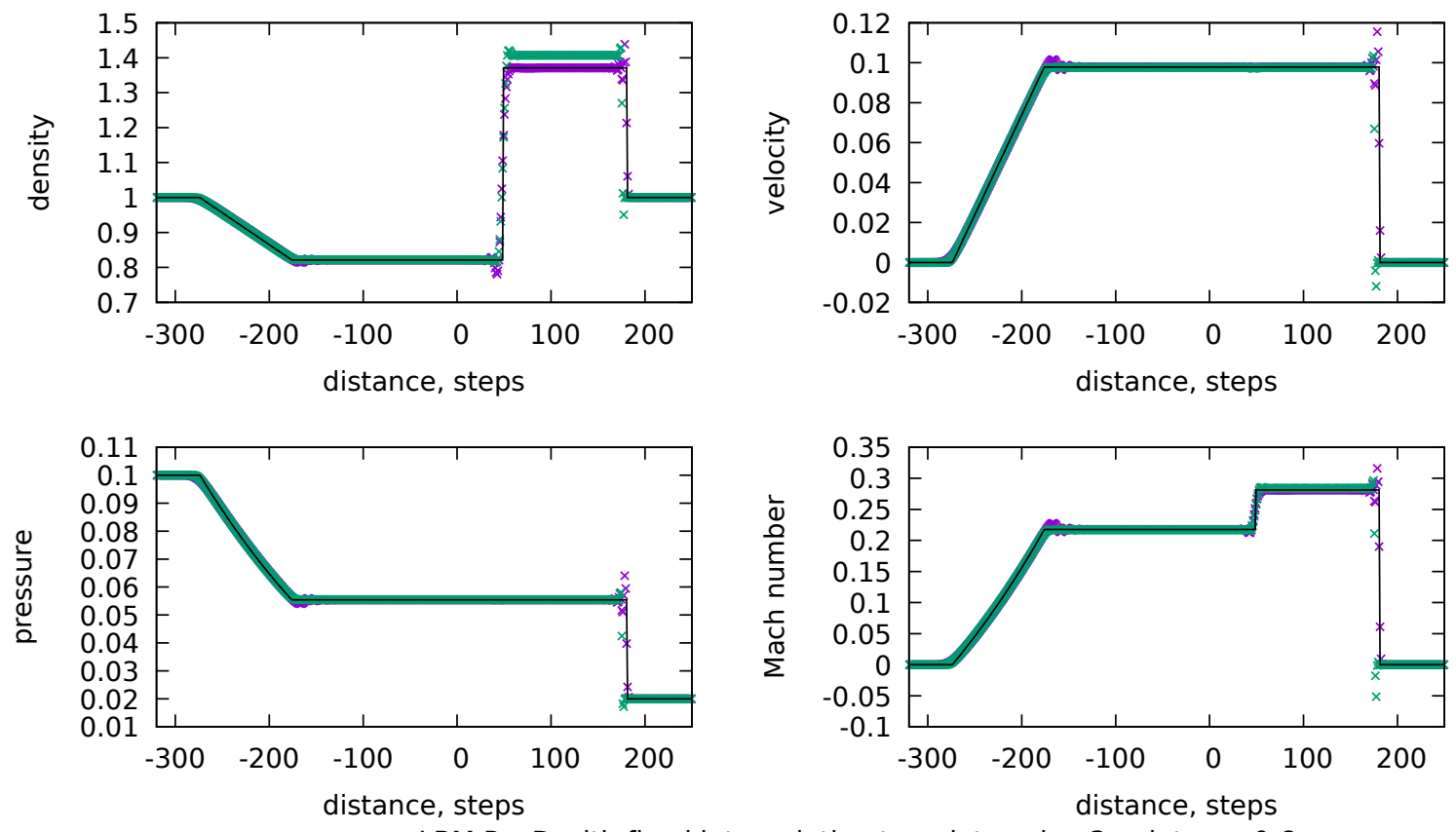

LBM PonD with fixed interpolation template using 3 points, $\tau=0.6$

LBM PonD with interpolation template using 4 points, $\tau=0.6$

Figure 6: Blast wave problem test at $500 \Delta t$

But for the non-fixed interpolation stencil the solution becomes unstable and the predictor-corrector iteration procedure doesn't converge.

We also demonstrate how PonD method handles with one more Riemann task with the following initial conditions:

$$
\begin{array}{cl}
\left.\rho\right|_{x<0}=1, & \left.\rho\right|_{x \geq 0}=1 ; \\
\left.P\right|_{x<0}=0.1, & \left.P\right|_{x \geq 0}=0.02 \\
\left.u\right|_{x<0}=0, & \left.u\right|_{x \geq 0}=0 .
\end{array}
$$

The solutions are shown in Fig. 6 .

It is evident from the density plot that the non-fixed interpolation stencil gives incorrect result - the density behind the shock wave is visibly higher than accurate value. The scheme with the fixed interpolation stencil gives the proper absolute value.

The analysis of the oscillatory nature of the one-dimensional Riemann tasks solution invites the suggestion to apply some kind of limiters to the PonD LBM scheme. Nevertheless, as it was previously shown, it is not possible to combine easily the variable stencil with conservativity of the PonD numerical scheme. Perhaps the reformulation of the scheme with fluxes could help to develop the use of limiters with controlling the conservativity. 


\subsection{Spherical Sod problem}

We have simulated the 3D spherical Sod problem with the following initial conditions:

$$
\begin{aligned}
\left.\rho\right|_{r<50}=1, & \left.\rho\right|_{r \geq 50}=0.125 ; \\
\left.P\right|_{r<50}=5 \cdot 10^{-2}, & \left.P\right|_{r \geq 50}=5 \cdot 10^{-3} ; \\
\left.u\right|_{r<50}=0, & \left.u\right|_{r \geq 50}=0,
\end{aligned}
$$

where $r=\sqrt{x^{2}+y^{2}+z^{2}}$.

Relaxation time was set to $\tau=0.6$. A regular 3D mesh was used for one eighth part of the whole spherical region. The simulated region is $0<x<$ $120,0<y<120,0<z<120$ and space steps are $\Delta x=\Delta y=\Delta z=\Delta r=1$. The mirror boundary conditions is applied on all boundaries, where unknown distribution function $f_{i}$ at negative $x, y$ or $z$-coordinates is reconstructed from the known function obtained by reflection symmetry.

We also compare the solution with the one obtained by the Godunov-type solver in spherical coordinates [16]. Space step resolution for the reference solution was $0.012 \Delta r$.

The problem is solved with the PonD D3Q125 method with two types of interpolation stencils: the fixed interpolation stencil for all cells using nearestneighbours points (second-order by use of $3 \times 3 \times 3=27$ points), and the flexible third-order interpolation stencil, that may vary for each of 125 distribution functions (tri-cubical interpolation based on $4 \times 4 \times 4=64$ points).

The density and Mach number cross-sections at different locations and several time instants are plotted in Fig. 7.

In Fig. 8, the solution is shown at the time step $t=150$ for different directions from the sphere center. The result for the non-fixed interpolation stencil looks also satisfactory despite the loss of the conservativity.

All calculations were performed with double precision. Maximum number of iteration steps was set to 100. Near the shock wave this number has been achieved, which shows that the convergence did not occur.

\section{Conclusion}

In this work, we have investigated the new and promising scheme PonD for fluid dynamic simulation.

We emphasize the two shortcomings of the originally published PonD D1Q3 method: the superfluous complexity of the streaming step, and loss of mass conservation.

We have found that in case the interpolation stencils for all $f_{i}$ in one streaming operation coincide, the streaming step may be written as an explicit update 


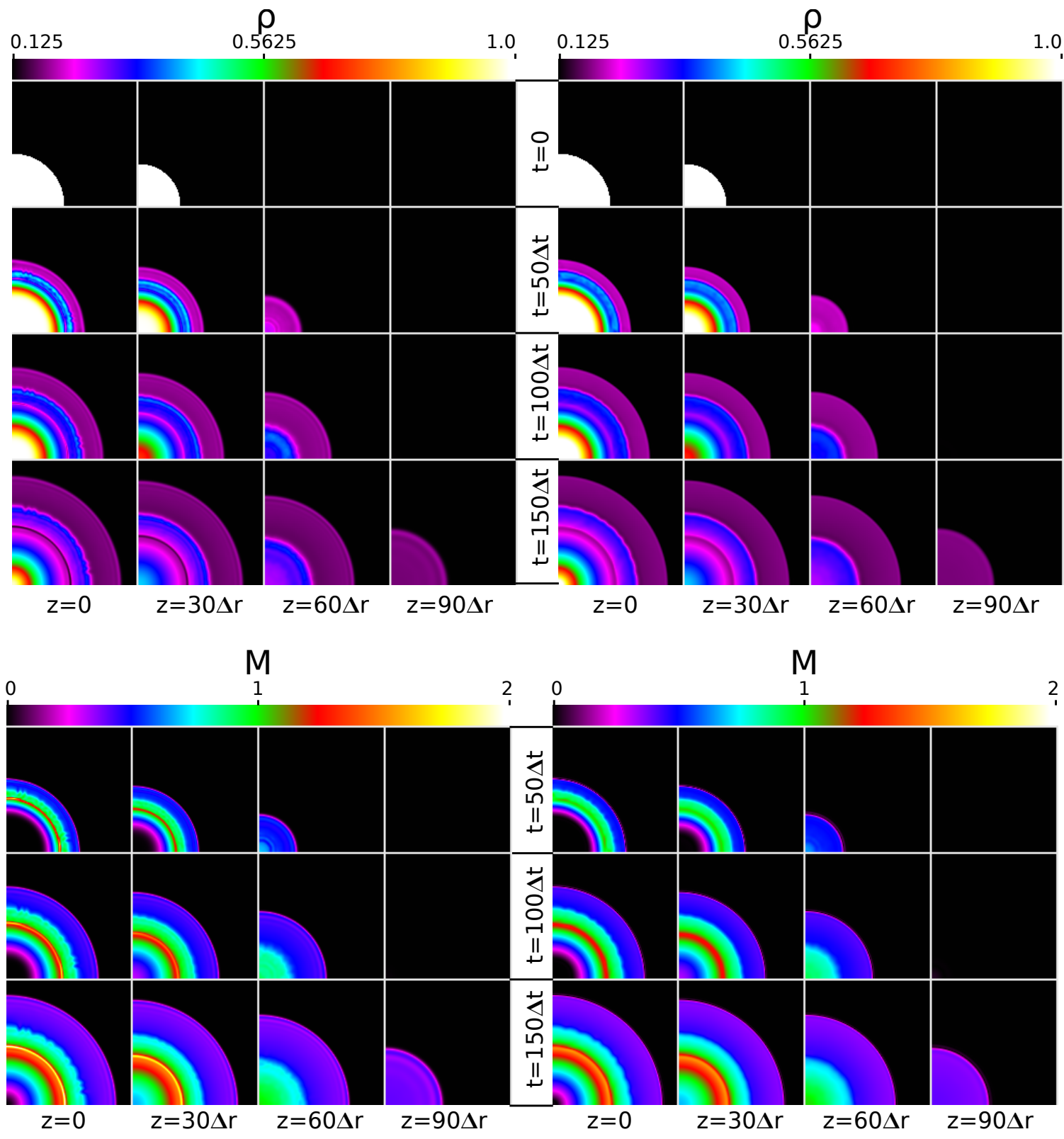

Figure 7: Spherical Sod problem solution. Top: the density cross-sections with different $\mathrm{z}$-coordinate at different time instants $=0$, 50, 100 and 150. Bottom: the Mach number cross-sections with different $z$-coordinate at different time instants $=50,100$ and 150. In the left half the fixed interpolation over 27 points is used; and in the right part the interpolation stencil is not fixed and uses $4 \times 4 \times 4$ points. 

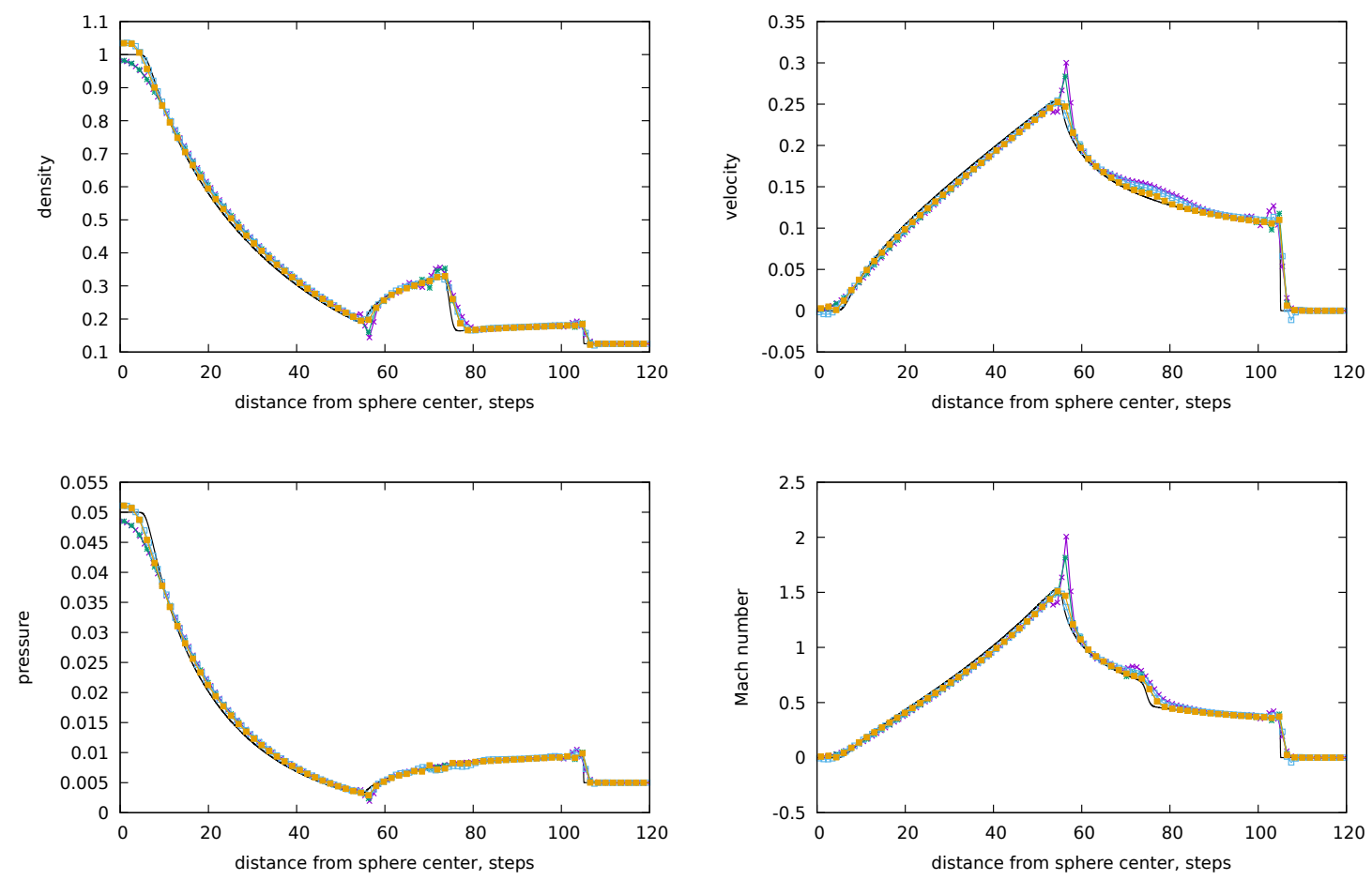

LBM PonD D3Q125 with 27-points rigid interpolation stencil (direction $=(1,0,0)$ ) - LBM PonD D3Q125 with 27-points rigid interpolation stencil (direction $=(1,1,1)$ ) LBM PonD D3Q125 with 64-points interpolation stencil (direction $=(1,0,0))$
LBM PonD D3O125 with 64-points interpolation stencil (direction $=(1,1,1))$

Figure 8: Spherical Sod problem solution at $150 \Delta t$

of several moments. If these moments include $\mathbf{u}$ and $T$, the gauge after the streaming step is known and can be explicitly derived from the corresponding $\rho, \mathbf{u}$ and $T$ of the nearest points and the predictor-corrector iterations are unnecessary.

Adjustment of the alternation of the scheme's stencil allows controlling the locations of the error sources. For example, the fixed interpolation stencil for the whole simulation domain makes the scheme conservative if enough number of the moments can be constructed, such as in the case of D1Q5 for one-dimensional case and D3Q125 for three-dimensional.

On the other hand, we see the PonD scheme as a valuable development in fluid dynamics simulation. The main finding of the original paper [1] is the method of gauge-transformation of the scheme variables. First of all, by putting the individual reference frame into each node of the computation grid some restrictions of the LBM is eliminated, because the second moment and therefore the temperature turn out to be correct in PonD even in nonisothermal flows. Secondly, by extracting the explicit scheme for $R$ moments we can view the PonD scheme as a variation of hydrodynamic finite-volume scheme, in which the information of the higher moments is not lost but retained in the remaining $(Q-R)$ DDFs. Thirdly, since the unlike LBM we do not require 
$e_{i}$ to be defined by the grid steps, we may choose Gauss-Hermite quadrature with higher precision.

In any case, we see that the scheme readily solves even the shock-wave problems with good precision. We see the new scheme as a raw idea that may be further developed and become a new trend in CFD. However for a complete success, it is necessary to develop conservative schemes or control the error of the nondissipative variables (mass, momentum and energy).

Aknowledgement. The work is supported by Russian Science Foundation, grant \# 18-71-10004.

\section{References}

[1] Benedikt Dorschner, Fabian Bösch, and Ilya V Karlin. Particles on demand for kinetic theory. Physical review letters, 121(13):130602, 2018.

[2] Sauro Succi. The lattice Boltzmann equation: for fluid dynamics and beyond. Oxford university press, 2001.

[3] Xiaowen Shan, Xue-Feng Yuan, and Hudong Chen. Kinetic theory representation of hydrodynamics: a way beyond the navier-stokes equation. Journal of Fluid Mechanics, 550:413-441, 2006.

[4] Paulo C Philippi, Luiz A Hegele Jr, Luís OE Dos Santos, and Rodrigo Surmas. From the continuous to the lattice boltzmann equation: The discretization problem and thermal models. Physical Review E, 73(5):056702, 2006.

[5] Andreas Krämer, Knut Küllmer, Dirk Reith, Wolfgang Joppich, and Holger Foysi. Semi-lagrangian off-lattice boltzmann method for weakly compressible flows. Physical Review E, 95(2):023305, 2017.

[6] Misun Min and Taehun Lee. A spectral-element discontinuous galerkin lattice boltzmann method for nearly incompressible flows. Journal of Computational Physics, 230(1):245-259, 2011.

[7] Marek Krzysztof Misztal, Anier Hernandez-Garcia, Rastin Matin, Henning Osholm Sørensen, and Joachim Mathiesen. Detailed analysis of the lattice boltzmann method on unstructured grids. Journal of Computational Physics, 297:316-339, 2015.

[8] Olga Filippova and Dieter Hänel. Grid refinement for lattice-bgk models. Journal of Computational physics, 147(1):219-228, 1998. 
[9] Abbas Fakhari and Taehun Lee. Finite-difference lattice boltzmann method with a block-structured adaptive-mesh-refinement technique. Physical Review E, 89(3):033310, 2014.

[10] Nianzheng Cao, Shiyi Chen, Shi Jin, and Daniel Martinez. Physical symmetry and lattice symmetry in the lattice boltzmann method. Physical Review E, 55(1):R21, 1997.

[11] Seung Hyun Kim, Heinz Pitsch, and Iain D Boyd. Accuracy of higherorder lattice boltzmann methods for microscale flows with finite knudsen numbers. Journal of computational physics, 227(19):8655-8671, 2008.

[12] Frank J Alexander, Hudong Chen, Shiyi Chen, and GD Doolen. Lattice boltzmann model for compressible fluids. Physical Review A, 46(4):1967, 1992.

[13] JM Buick and JA Cosgrove. Investigation of a lattice boltzmann model with a variable speed of sound. Journal of Physics A: Mathematical and General, 39(44):13807, 2006.

[14] Gary A Sod. A survey of several finite difference methods for systems of nonlinear hyperbolic conservation laws. Journal of computational physics, 27(1):1-31, 1978.

[15] Paul Woodward and Phillip Colella. The numerical simulation of twodimensional fluid flow with strong shocks. Journal of computational physics, 54(1):115-173, 1984.

[16] Eleuterio F Toro. Riemann solvers and numerical methods for fluid dynamics: a practical introduction. Springer Science \& Business Media, 2013. 